\title{
Changes in the relationship between the right internal jugular vein and an anatomical landmark after head rotation
}

\author{
Sun Young Park, Min Jung Kim, Mun Gyu Kim, Se Jin Lee, Sang Ho Kim, Si Young Ok, \\ and Soon Im Kim
}

Department of Anesthesiology and Pain Medicine, Soonchunhyang University Hospital, Seoul, Korea

Background: This study was performed to ultrasonographically demonstrate the changes in relationship between the right internal jugular vein (IJV) and an anatomical landmark in two different head positions: neutral and rotated.

Methods: This was a randomized clinical trial. One hundred patients scheduled for elective surgery under general anesthesia with endotracheal intubation were enrolled in this study. The patients were placed in the supine position with a neutral head position and without a pillow. The apex of the triangle formed by the sternal and clavicular heads of the sternocleidomastoid muscle and clavicle was marked (AL point: anatomical landmark point). Ultrasonography of the neck anatomy was performed and the skin was marked at the central point of the IJV (US point: ultrasonography point). The other investigator measured the distance from the AL point to the US point (ALUS distance). The patient's head was then turned $30^{\circ}$ to the left; the same procedure was repeated and the AL-US distance was again measured. The changes in AL-US distance were calculated.

Results: The AL-US distance increased significantly after $30^{\circ}$ head rotation compared with that in a head neutral position. The mean \pm SD of the AL-US distance was $0.28 \pm 0.78 \mathrm{~cm}$ in the neutral head position and $0.83 \pm 1.03 \mathrm{~cm}$ in the head rotated position.

Conclusions: The anatomical landmark point becomes more distant from the actual right IJV point and moves more medially after head rotation. We suggest minimizing the angle of head rotation and taking this distance into consideration when using the landmark-guided method. (Korean J Anesthesiol 2011; 61: 107-111)

Key Words: Anatomy, Jugular vein, Ultrasonography.

\footnotetext{
Received: December 7, 2010. Revised: January 12, 2011. Accepted: January 15, 2011.

Corresponding author: Sun Young Park, M.D., Department of Anesthesiology and Pain Medicine, Soonchunhyang University Hospital, 657, Hannam-dong, Yongsan-gu, Seoul 140-743, Korea. Tel: 82-2-709-9291, Fax: 82-2-790-0394, E-mail: sunnypark97@gmail.com

(c) This is an open-access article distributed under the terms of the Creative Commons Attribution Non-Commercial License (http:// creativecommons.org/licenses/by-nc/3.0/), which permits unrestricted non-commercial use, distribution, and reproduction in any medium, provided the original work is properly cited.
} 


\section{Introduction}

The right internal jugular vein (IJV) is frequently used as a central venous route due to its short, straight course to the superior vena cava. It has a consistent, predictable anatomical location, and readily identifiable and palpable surface landmarks.

Most landmark-guided methods for cannulation of the IJV dictate that the head should be turned away from the side of the neck being entered $[1,2]$. The effect of head rotation on the position of the IJV relative to the common carotid artery (CCA) has been demonstrated [3-6]. As the head is rotated away from the midline, the IJV becomes more directly anterior to the CCA, resulting in a greater overlap of the IJV and CCA as compared with a neutral head position.

Although the general anatomy and relationships of the IJV with the landmark are known, there are possible variations in different head positions, as shown in the relationship with the CCA. Anatomical variation and the unreliability of external landmarks have been implicated as the cause of difficulties in cannulation. Knowledge of the anatomy of the IJV and its variations may improve the success and safety of puncture [7]. We hypothesized that the rotation of head would change the relationship between the right IJV and an anatomical landmark. The present study was performed to ultrasonographically demonstrate the changes in relationship between the right IJV and the anatomical landmark in two different head positions: neutral and rotated.

\section{Materials and Methods}

This study was approved by the Institutional Review Board and registered with ClinicalTrials. gov (NCT01168453). This was a randomized clinical trial. After obtaining written informed consent, 100 patients weighing $40-100 \mathrm{~kg}$ and with heights of $140-190 \mathrm{~cm}$, who were between 18 and 80 years of age and scheduled for elective surgery under general anesthesia with endotracheal intubation, were enrolled in the study between
May 2009 and February 2010. Exclusion criteria included a history of neck surgery or trauma involving the neck, previous IJV-related interventions (e.g., venous catheterization), limitation of neck movement, and a body mass index $\geq 30$. If the anatomical landmark was not easily discernable, the patient was eliminated from the analysis. Patient characteristics are shown in Table 1.

After induction of general anesthesia, all patients were tracheally intubated and their lungs were mechanically ventilated using pressure control mode with a peak pressure of 15-20 mmHg without positive end expiratory pressure (PEEP); the respiratory rate was $10-12$ breaths per minute. The patients were placed in the supine position with a neutral head position and without a pillow. The operating table was placed in a level position in the transverse and longitudinal planes. The apex of the triangle formed by the sternal and clavicular heads of the sternocleidomastoid muscle and clavicle was marked by one experienced anesthesiologist (AL point). Two-dimensional ultrasonography of the neck anatomy was then performed using a 13-6 MHz $25 \mathrm{~mm}$ broadband linear array probe and portable ultrasound machine (MicroMaxx ${ }^{\circledR}$, Sonosite Inc., Bothell, WA, USA). The probe was placed at an angle of approximately $90^{\circ}$ on the patient's neck skin without significant compression to maintain the normal shape and location of the vessel. The head of the probe was placed parallel to the transverse plane, and the tip was placed medially (Fig. 1). A second investigator placed the center of the probe at the AL point and evaluated whether the central perpendicular line of the image passed through the right IJV. If the line passed through the vein, it was scored as a "hit"; if not, it was scored as a "miss". The investigator then moved the probe medially or laterally to the AL point in the same transverse plane and fixed the probe when the center of the right IJV was at the center of the ultrasonography image. A mark was placed on the skin at the central point of the probe head (US point), and the depth of the right IJV center was measured. All of these procedures were performed during expiration. The other investigator, who was blinded to this study, measured the distances from the clavicle to the AL point,

Table 1. Patient Characteristics

\begin{tabular}{lccc}
\hline & $\begin{array}{c}\text { All patients } \\
(\mathrm{n}=100)\end{array}$ & $\begin{array}{c}\text { Men } \\
(\mathrm{n}=53)\end{array}$ & $\begin{array}{c}\text { Women } \\
(\mathrm{n}=47)\end{array}$ \\
\hline Age $(\mathrm{yr})$ & $53.4(18-79)$ & $53.3(18-79)$ & $53.4(18-70)$ \\
Weight $(\mathrm{kg})$ & $63.6 \pm 10.8$ & $67.9 \pm 9.9$ & $58.8 \pm 9.9$ \\
Height $(\mathrm{cm})$ & $162.5 \pm 8.2$ & $167.5 \pm 6.3$ & $156.8 \pm 6.0$ \\
BMI $\left(\mathrm{kg} / \mathrm{m}^{2}\right)$ & $24.0 \pm 3.2$ & $24.1 \pm 3.2$ & $23.8 \pm 3.3$ \\
Distance from clavicle to AL $(\mathrm{cm})$ & $4.0 \pm 0.6$ & $4.1 \pm 0.6$ & $3.9 \pm 0.6$ \\
Distance from midline to AL $(\mathrm{cm})$ & $5.7 \pm 1.0$ & $6.1 \pm 1.0$ & $5.3 \pm 0.7$ \\
Depth of right IJV center $(\mathrm{cm})$ & $1.3 \pm 0.2$ & $1.3 \pm 0.2$ & $1.2 \pm 0.2$ \\
\hline
\end{tabular}

Data are given as the mean (range) or mean \pm SD. BMI: body mass index, AL: anatomical landmark point, IJV: internal jugular vein. 
from the midline of the neck to the AL point, and from the AL point to the US point (AL-US distance). The distance between the two points was considered "positive" if the US point was lateral to the AL point and "negative" if it was medial to the AL point. After these procedures, the patient's head was turned $30^{\circ}$ (between the perpendicular line and patients' noses) to the left using a semicircular leveled protractor. The AL point was reevaluated and adjustments were made if necessary. The second investigator placed the center of the probe at the AL point and reevaluated whether the central perpendicular line of the image passed through the right IJV, marked the US point, and again the other investigator measured the distance from the AL point to the US point. The change in AL-US distances was

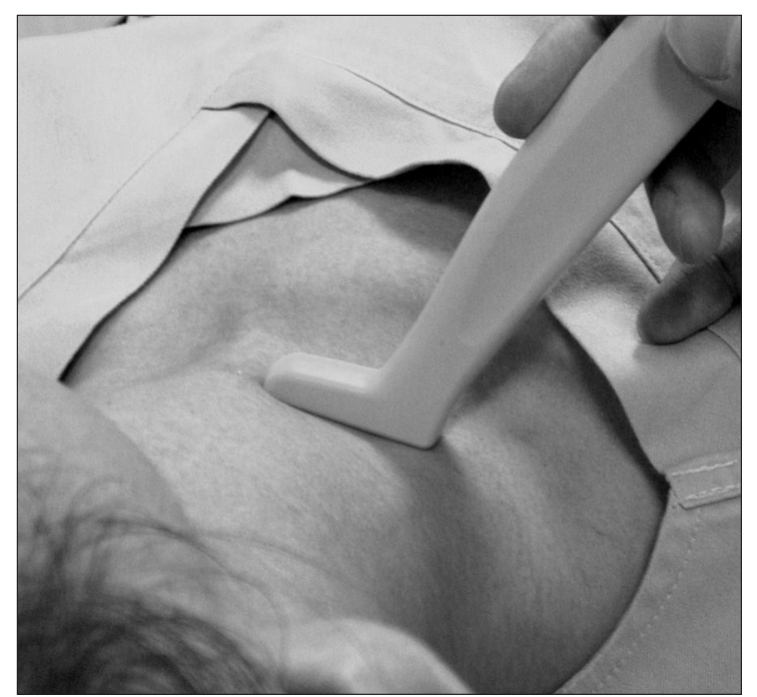

Fig. 1. The probe position on a patient's neck. The head of the probe was placed parallel to the transverse plane, with the tip placed medially.

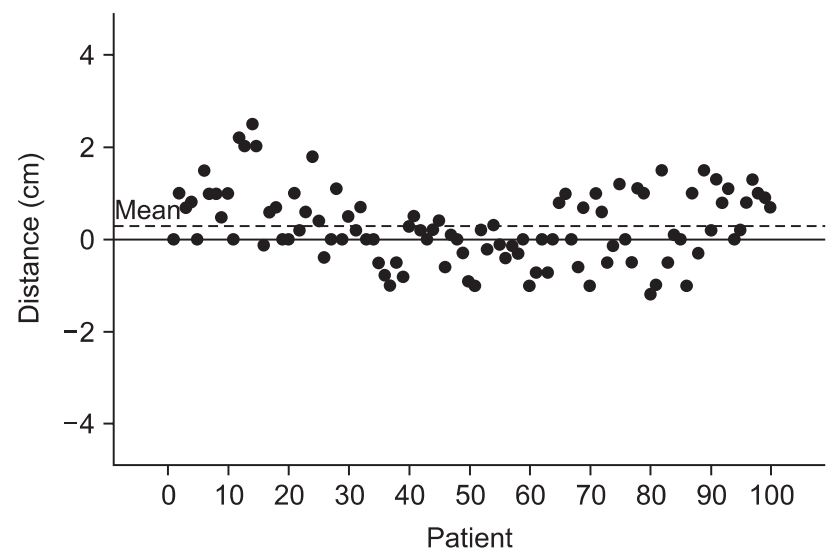

Fig. 2. The distance from AL to US point in the neutral head position. The dashed-dotted line represents the mean value. AL: anatomical landmark point, US point: ultrasonography point. calculated by the AL-US distance in the head-rotated position minus the distance in the neutral position. A paper ruler attached to the skin along the curvature was used to measure the distances.

The primary outcome variable was the distance between the AL and US points. The independent variables were head position, age, gender, and BMI. BMI was calculated as weight $(\mathrm{kg}) /$ height $^{2}\left(\mathrm{~m}^{2}\right)$. The data were expressed as number of patients, mean (range) or means \pm SD. A comparative study was performed of the AL-US distance between neutral head position and rotation position, and for age, gender, and BMI. The statistical significance of the changes in AL-US distance at different positions within the same patient was analyzed using a paired $t$ test. The correlation of independent variables with the AL-US distance was determined by linear regression analysis. Fisher's exact test was used to compare the proportion of "misses" by head position. Data were analyzed using SPSS for Windows (version 13.0; SPSS Inc., Chicago, IL).

\section{Results}

IJVs were easily visualized with ultrasound, and the anatomical landmark was readily discernable in all patients. Data were collected for all 100 patients enrolled in the study. Enrolled patients varied in age from 18 to 79 years, and the study population consisted of 53 males and 47 females. The average depth of the right IJV was $1.3 \pm 0.2 \mathrm{~cm}$ (maximum, $2 \mathrm{~cm}$ ).

The mean \pm SD of the AL-US distance was $0.28 \pm 0.78 \mathrm{~cm}$ in the neutral head position and $0.83 \pm 1.03 \mathrm{~cm}$ in the head rotated position (Fig. 2 and 3). The change was statistically significant $(0.54 \pm 0.50 \mathrm{~cm}, 95 \% \mathrm{CI} 0.44$ to $0.64, \mathrm{P}<0.001)$. Linear regression analysis indicated that age, gender, and BMI were not significant. The proportion of "misses" increased significantly after $30^{\circ}$ head

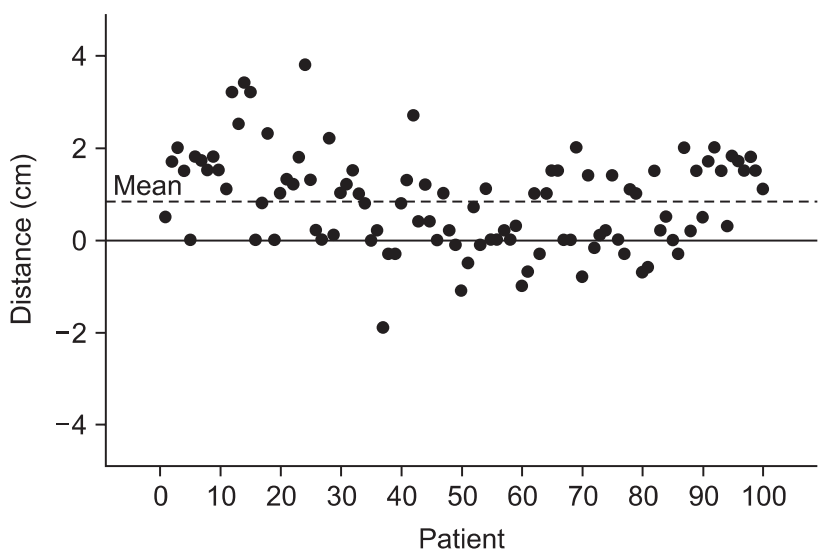

Fig. 3. The distance from AL to US point at a $30^{\circ}$ head rotation position. The dashed-dotted line represents the mean value. AL: anatomical landmark point, US point: ultrasonography point. 
rotation compared with that in a neutral position ( $24 \%$ vs. $11 \%$, $\mathrm{P}<0.001)$.

\section{Discussion}

There was a significant change in the relationship between the right IJV and the anatomical landmark in the two different head positions. The AL-US distance increased significantly after $30^{\circ}$ head rotation compared with that in a head neutral position. When we assume the US point as an actual right IJV point, this indicated that the anatomical landmark point (AL point) became more distant from the actual right IJV point, and that the AL point moved more medially relative to the actual point after head rotation.

Although this change may seem small $(0.54 \pm 0.50 \mathrm{~cm}, 95 \%$ CI 0.44 to 0.64 ), it should be taken into consideration. As the anatomical landmark point becomes more distant from the actual right IJV point, head rotation may increase the failure rate of right IJV catheterization when the landmark-guided method is used. The "miss" rate increased after head rotation in this study. Furthermore, after head rotation, the AL point was placed more medial to the US point than when in the neutral position. As the carotid artery is frequently placed medially to the right IJV [8,9], the risk of carotid puncture may be increased.

Bailey et al. [7] found the central landmark approach to have a bias toward a position medial to the center of the right IJV and to be imprecise. This was consistent with our findings. However, in their study, the mean bias was $0.37 \mathrm{~cm}$ (95\% CI, $0.27-0.48$ ) and 2SD was $1.05 \mathrm{~cm}$ when the patient's head was turned $30-35^{\circ}$. In the present study, the bias (AL-US distance) was $0.83 \pm 1.03 \mathrm{~cm}$ with $30^{\circ}$ rotation of the head. This may have been due to differences in probe angle to the skin and the method of distance measurement used. In their study, the probe was aimed toward the ipsilateral nipple at a $30^{\circ}$ angle to the coronal plane to simulate the needle path and measure the length of a perpendicular line from the center of the IJV to the midline cursor on the ultrasound images. However, we placed the probe almost perpendicular to the skin to obtain images just beneath the landmark and measure the distance at the skin. The difference in the angle relative the sagittal plane may explain why the distance here was almost twice that in the previous study. And it may also explain the high incidence of "miss" rate in both head position in this study. This result confirms the benefit of needle lateralization when using the landmark-guided method with the head turned to the contralateral side. In addition, we obtained the same result, that the landmark was positioned medially to the IJV, which also supports the suggestion that the needle should be redirected laterally when the first needle pass is not successful [7], unlike the recommendation of McGee and Gould [10]. As the head is rotated, the surface area may rotate more than the inner area, and this induces a medial shift in the landmark point compared with the IJV.

Troianos et al. [5] measured the positional relationship between the IJV and CCA using ultrasound imaging and magnetic resonance imaging. When patients were placed in a position with the head turned to the contralateral side, there was often overlap between the IJV and CCA. There seemed to be hardly any overlap if the head was in the neutral position. Sulek et al. [6] also demonstrated that contralateral head rotation alters the anatomical relationship of the IJV and CCA. They advocated neutral or near-neutral head positioning during IJV cannulation to reduce the risk of carotid artery puncture associated with the overlap of the two vessels caused or increased by head rotation. Like the positional alteration between the IJV and CCA, we observed a change in the relationship between the IJV and the anatomical landmark after head rotation. As head rotation makes the two points more distant, the neutral position could increase the accuracy of the landmark. The landmark-guided method with a neutral head position has been highly successful with few complications during IJV cannulation [11].

Although head rotation positioning technique is used usually because it facilitate expose and approach to IJV, the neutral head position technique is advantageous in some types of case, especially in spine injury patients. Head rotation itself may lead to reduction of internal jugular vein blood flow, and therefore the neutral position appears to be the preferred alignment for a more distended vein $[4,11]$. This position is also advantageous for the positional relationship between the right IJV and the CCA, and the anatomical landmark as explained above. One thing to be considered is that the mean AL-US distance was $0.28 \pm 0.78 \mathrm{~cm}$ with a neutral head position. Therefore, needle lateralization may not be necessary when using the landmarkguided method with a neutral head position. Instead, a more acute needle insertion angle is required because the needle entry point of this landmark-guided method is expected to be almost directly above the IJV, and for avoiding the mandible angle. Therefore, we suggest that the needle should be maintained at an angle of $60-90^{\circ}$ above the coronal plane with the direction parallel to the sagittal plane when the landmarkguided method is used with a neutral head position. This approach is similar to the method of Willeford and Reitan [11], although the entry point differs. Lieberman et al. [12] held the probe within a parasagittal plane and directed it $30^{\circ}$ caudad, and the incidence of an ultrasound beam intersecting the IJV was $<20 \%$ in the neutral position. The $30^{\circ}$ angle may have been responsible for the low incidence.

The average depth of the right IJV was $1.3 \pm 0.2 \mathrm{~cm}$ with a maximum of $2 \mathrm{~cm}$ among the 100 patients in this study. 
McGee and Gould [10] also reported that the IJV is generally encountered approximately $1.3 \mathrm{~cm}$ under the skin. The average skin-to-vein distance, as traveled by a cannulation needle at an approximately $30^{\circ}$ angle to the skin for 314 IJVs measured, was $2.59 \pm 0.45 \mathrm{~cm}$ in the previous study [2]. Therefore, it is not necessary to use a longer finder needle than $3 \mathrm{~cm}$ in most patients, and needle advancement greater than this depth would be unnecessary and also dangerous; although, the depth can vary among individual patients depending on regional adiposity.

It would be ideal to use ultrasound when attempting cannulation [13,14]; however, ultrasound is not always available. When ultrasound devices are not available, it is necessary to rely on the landmark-guided technique for central venous cannulation. Therefore, knowledge of the anatomical locations and variations in position of the IJV is important for physicians. The results of this study may facilitate decision making regarding the needle entry point, angle, and head position during landmark-guided IJV cannulation.

This study has several limitations. The anatomical relationship described in this study was in the cross-sectional plane, and not in the traditional directional plane of the cannulation needle. This makes it difficult to extend our findings to techniques recommending that needle insertion be directed laterally or toward the ipsilateral nipple with an angle of $30^{\circ}$ to $45^{\circ}$. However, our findings may help physicians to speculate on the location and changes in the location of the right IJV under the skin in the two head positions. This should be considered during attempts to cannulate the IJV without imaging devices.

And the SD of AL-US distances were large relative to mean values. This is caused by the inaccuracy of landmark to represent the location of IJV. It could weaken the statistical significance our findings. However, we considered that the significant change of the AL-US distances is still useful information clinically.

In conclusion, the anatomical landmark point becomes more distant from the actual right IJV point and moved more medially after head rotation. We suggest the angle of head rotation should be minimized and this distance should be taken into consideration when using the landmark-guided method.

\section{References}

1. Daily PO, Griepp RB, Shumway NE. Percutaneous internal jugular vein cannulation. Arch Surg 1970; 101: 534-6.

2. Metz S, Horrow JC, Balcar I. A controlled comparison of techniques for locating the internal jugular vein using ultrasonography. Anesth Analg 1984; 63: 673-9.

3. Kim SI, Kang JH, Baek Y, Kim SH, Ok S, Kim SC. The influence of head rotation on the anatomical relationship of the right internal jugular vein and the carotid artery. Korean J Anesthesiol 2008; 55: 538-42.

4. Bazaral M, Harlan S. Ultrasonographic anatomy of the internal jugular vein relevant to percutaneous cannulation. Crit Care Med 1981; 9: 307-10.

5. Troianos CA, Kuwik RJ, Pasqual JR, Lim AJ, Odasso DP. Internal jugular vein and carotid artery anatomic relation as determined by ultrasonography. Anesthesiology 1996; 85: 43-8.

6. Sulek CA, Gravenstein N, Blackshear RH, Weiss L. Head rotation during internal jugular vein cannulation and the risk of carotid artery puncture. Anesth Analg 1996; 82: 125-8.

7. Bailey PL, Whitaker EE, Palmer LS, Glance LG. The accuracy of the central landmark used for central venous catheterization of the internal jugular vein. Anesth Analg 2006; 102: 1327-32.

8. Turba UC, Uflacker R, Hannegan C, Selby JB. Anatomic relationship of the internal jugular vein and the common carotid artery applied to percutaneous transjugular procedures. Cardiovasc Intervent Radiol 2005; 28: 303-6.

9. Newton JD, Sprigings DC. Central venous catheterization. N Engl J Med 2007; 357: 943.

10. McGee DC, Gould MK. Preventing complications of central venous catheterization. N Engl J Med 2003; 348: 1123-33.

11. Willeford KL, Reitan JA. Neutral head position for placement of internal jugular vein catheters. Anaesthesia 1994; 49: 202-4.

12. Lieberman JA, Williams KA, Rosenberg AL. Optimal head rotation for internal jugular vein cannulation when relying on external landmarks. Anesth Analg 2004; 99: 982-8.

13. Denys BG, Uretsky BF, Reddy PS. Ultrasound-assisted cannulation of the internal jugular vein. A prospective comparison to the external landmark-guided technique. Circulation 1993; 87: 1557-62.

14. Lamperti M, Cortellazzi P, D'Onofrio G, Subert M, Falcone C, Filippini G, et al. An outcome study on complications using routine ultrasound assistance for internal jugular vein cannulation. Acta Anaesthesiol Scand 2007; 51: 1327-30. 